\title{
Thermoelectric DC conductivities from black hole horizons
}

\author{
Aristomenis Donos ${ }^{a}$ and Jerome P. Gauntlett ${ }^{b}$ \\ ${ }^{a}$ DAMTP, University of Cambridge, \\ Cambridge, CB3 0WA, U.K. \\ ${ }^{b}$ Blackett Laboratory, Imperial College, \\ London, SW7 2AZ, U.K. \\ E-mail: a.donos@damtp.cam.ac.uk, j.gauntlett@imperial.ac.uk
}

AbStract: An analytic expression for the DC electrical conductivity in terms of black hole horizon data was recently obtained for a class of holographic black holes exhibiting momentum dissipation. We generalise this result to obtain analogous expressions for the DC thermoelectric and thermal conductivities. We illustrate our results using some holographic Q-lattice black holes as well as for some black holes with linear massless axions, in both $D=4$ and $D=5$ bulk spacetime dimensions, which include both spatially isotropic and anisotropic examples. We show that some recently constructed ground states of holographic Q-lattices, which can be either electrically insulating or metallic, are all thermal insulators.

KEYworDs: Gauge-gravity correspondence, Black Holes in String Theory, AdS-CFT Correspondence, Holography and condensed matter physics (AdS/CMT)

ARXIV EPRINT: 1406.4742 


\section{Contents}

1 Introduction 1

2 The holographic black holes $\quad 4$

2.1 The Holographic models 4

2.2 The black hole backgrounds 5

3 Calculating the DC conductivities $\quad 6$

3.1 Calculating $\sigma$ and $\bar{\alpha} \quad 6$

3.2 Calculating $\alpha$ and $\bar{\kappa} \quad 9$

3.3 Comments 11

4 Examples $\quad 13$

$\begin{array}{lll}4.1 & \text { Models with massless axions } & 14\end{array}$

$\begin{array}{lll}\text { 4.1.1 Analytic isotropic solutions } & 14\end{array}$

4.1.2 Anisotropic neutral black holes in $D=5 \quad 14$

$\begin{array}{lll}4.2 & \text { Holographic Q-lattices } & 15\end{array}$

4.2.1 Q-lattice black holes with $A d S_{2} \times \mathbb{R}^{2}$ in the IR at $T=0 \quad 15$

$\begin{array}{ll}\text { 4.2.2 The Q-lattice black holes of }[6] & 16\end{array}$

$\begin{array}{llr}5 & \text { Final comments } & 17\end{array}$

$\begin{array}{lr}\text { A Killing vector identity } & 19\end{array}$

B Holographic renormalisation and the heat current $\quad 19$

$\begin{array}{lll}\text { B.1 A complementary point of view } & 21\end{array}$

C DC transport from linear sources in time $\quad 22$

\section{Introduction}

A striking feature of holography is that it provides a prescription for calculating transport coefficients of strongly coupled systems by analysing small perturbations about the black holes that describe the equilibrium state. In this paper we will be interested in strongly coupled CFTs at finite temperature, $T$, and chemical potential, $\mu$, with respect to an abelian global symmetry, which are described by asymptotically AdS black holes which are electrically charged if $\mu \neq 0$. The addition of a small electric field $E_{i}$ and/or thermal gradient $\nabla_{i} T$ will induce an electric current $J^{i}$ and a heat current $Q^{i}=T^{t i}-\mu J^{i}$, where $T^{a b}$ 
is the stress tensor and $i$ labels a spatial index. At linearised order we have the generalised Ohm/Fourier law:

$$
\left(\begin{array}{c}
J \\
Q
\end{array}\right)=\left(\begin{array}{cc}
\sigma & \alpha T \\
\bar{\alpha} T & \bar{\kappa} T
\end{array}\right)\left(\begin{array}{c}
E \\
-(\nabla T) / T
\end{array}\right) .
$$

In this expression the matrix $\sigma$ is the electrical conductivity, $\alpha, \bar{\alpha}$ are the thermoelectric conductivities and $\bar{\kappa}$ is the thermal conductivity.

Much work has been done on obtaining the AC conductivities, particularly $\sigma$, in a variety of different contexts, by allowing for perturbations with a time dependence of the form $e^{-i \omega t}$ and then imposing ingoing boundary conditions at the black hole event horizon. These calculations, reviewed in $[1,2]$, utilise the fact that the conductivities are related to retarded Green's functions via $i \omega \sigma=G_{J J}^{R}(\omega)$ etc. The DC conductivities, if they are finite, can then be obtained by carefully taking the $\omega \rightarrow 0$ limit.

Here we want to directly calculate the DC response. It has been known for some time that for the case for translationally invariant CFTs with $\mu=0$ that are described by the AdS-Schwarzschild black brane solution [3], it is possible to obtain an expression for $\sigma_{D C}$ in terms of horizon data, somewhat analogous to the result for the shear viscosity $[4,5]$. However, when $\mu \neq 0$ a consideration of the standard AdS-RN black brane, describing a translationally invariant system, reveals that this is not always the case. Specifically, the translation invariance and finite charge density imply that the DC conductivities are infinite. More precisely, the real parts of the $\mathrm{AC}$ conductivities have a delta function at zero frequency.

Nonetheless, for holographic lattices associated with black holes with broken translation invariance, or more generally when there is a mechanism for momentum dissipation, the associated DC conductivities will be finite and one might hope to obtain a result in terms of black hole horizon data. This was recently confirmed for the electric conductivity $\sigma$ for a class of holographic Q-lattices in [6]. ${ }^{1}$ Such Q-lattices were introduced in [14] and break translation invariance periodically while preserving a homogeneous metric. This is a significant technical simplification since the black holes can be constructed by solving ODEs instead of PDEs ${ }^{2}$ as in the constructions [15, 18-22]. It was shown in [6] that the electrical conductivity $\sigma$ can be expressed in terms of horizon data and here we generalise this analysis to obtain analogous expressions for $\alpha, \bar{\alpha}$ and $\bar{\kappa}$. A key step in our derivation is to manipulate the bulk equations of motion to obtain expressions for the electric and heat currents that are independent of the holographic radial coordinate and hence can be evaluated at the black hole horizon.

For illustration we will mostly consider holographic Q-lattices in $D=4$ bulk dimensions which break translation invariance in either one or both of the two spatial directions of

\footnotetext{
${ }^{1}$ Similar results were obtained in $[7,8]$, using a different approach based on massive gravity, extending [911]. A related result for inhomogeneous lattices, for small lattice strength, was obtained in [12]. Very recently the methods of [6] were used to obtain the electrical DC conductivity in the presence of a magnetic field in [13].

${ }^{2}$ For the special case of $D=5$, helical lattices can also be constructed by solving ODEs [15], extending $[16,17]$.
} 
the dual CFT. In particular, our analysis will cover both isotropic and anisotropic cases. The matrices $\sigma, \alpha, \bar{\kappa}$ are diagonal and furthermore, since the backgrounds are time-reversal invariant, we have a symmetric conductivity matrix with $\bar{\alpha}=\alpha$. It is straightforward to generalise our analysis to holographic Q-lattices in other dimensions and indeed our final result can be cast in a $D$ independent manner. Our approach can also be extended to other holographic lattices, including inhomogeneous lattices [23].

Our results give the DC conductivities for all temperatures. They also provide a convenient tool to extract the low-temperature scaling behaviours of the DC conductivities when the far IR behaviour of the $T=0$ ground states are known. This allows one to determine, for example, if the ground state is exhibiting metallic or insulating behaviour with respect to the electric conductivity. Specifically, one can construct "small" black holes, by heating up the zero temperature IR ground states and then extract the low-temperature behaviour of the DC conductivity.

The low temperature scaling behaviour of the electrical conductivity was also discussed in [24] using the memory matrix formalism. The results here and in [6] complement and extend this illuminating work within a holographic setting. Specifically, the memory matrix formalism requires that the $T=0$ ground states are translationally invariant and that the strength of the lattice is small, whereas our approach has no such restrictions.

For the class of black holes that we consider we find, in general, that we can write

$$
\frac{\bar{\kappa}}{\alpha}=\frac{T s}{q}, \quad \bar{L} \equiv \frac{\bar{\kappa}}{\sigma T} \leq \frac{s^{2}}{q^{2}},
$$

where $s$ and $q$ are the entropy density and electric charge of the black holes, respectively. In situations where the memory matrix formalism applies, using the results of [25] we can rewrite these as

$$
\frac{\bar{\kappa}}{\alpha}=\frac{\chi_{Q P}}{\chi_{J P}}, \quad \bar{L} \leq \frac{1}{T^{2}} \frac{\chi_{Q P}^{2}}{\chi_{J P}^{2}},
$$

where in the notation of [25], $\chi$ are static susceptibilities involving the operators for the total momentum $P$, electric current $J$ and heat current $Q$.

We will illustrate our results for some known examples, including the isotropic and anisotropic Q-lattice black holes constructed in [6, 14]. These include solutions where, at $T=0$, the black holes approach $A d S_{2} \times \mathbb{R}^{D-2}$ in the far IR, perturbed by irrelevant operators. We obtain low-temperature scaling behaviours for the full DC conductivity matrix that are determined by the scaling dimension, $\Delta\left(k_{1}\right)$, of the least irrelevant IR operator, thus generalising the results of [24]. We find that

$$
\sigma \sim T^{2-2 \Delta\left(k_{1}\right)}, \quad \alpha \sim T^{2-2 \Delta\left(k_{1}\right)}, \quad \bar{\kappa} \sim T^{3-2 \Delta\left(k_{1}\right)} .
$$

with $\Delta\left(k_{1}\right)>1$. We see that while $\sigma$ and $\alpha$ will diverge at $T=0, \bar{\kappa}$ will go to zero, if $1<\Delta\left(k_{1}\right)<3 / 2$, a constant, if $\Delta\left(k_{1}\right)=3 / 2$, and diverge if $3 / 2<\Delta\left(k_{1}\right)$. We also calculate $\kappa \equiv \bar{\kappa}-\alpha^{2} T / \sigma$, the thermal conductivity at zero electric current, a quantity that is more readily measurable than $\bar{\kappa}$. Interestingly, we find that for these $A d S_{2} \times \mathbb{R}^{2}$ ground states we have $\kappa \sim T$ independent of $\Delta\left(k_{1}\right)$. 
The Q-lattice black hole solutions of $[6,14]$ also include black holes which at $T=$ 0 approach other IR solutions ${ }^{3}$ which break translation invariance and can be electrical insulators or metals depending on whether $\sigma$ is zero or non-zero respectively. Here we will find that the insulating ground states and, somewhat surprisingly, also the metallic ground states, are both thermal insulators with $\kappa, \bar{\kappa}=0$ at $T=0$. This suggests that the nonvanishing electrical conductivity of these metals can be thought of, loosely, as arising from the evolution of charged particle-hole pairs, possibly pair produced, in an electric field.

We also consider some other examples which involve linear massless axions which lead to momentum dissipation [8, 26-28] (see also [6, 7]). Although these solutions share some similarities with the Q-lattice black holes of $[6,14]$, they differ in several respects. While the Q-lattice is a periodic deformation of the UV physics by relevant operators of the CFT, the linear massless axion is a non-periodic deformation using marginal operators. In the case that the linear axion black hole solutions approach $A d S_{2} \times \mathbb{R}^{D-2}$ in the far IR, the linear axionic deformation is still present, in contrast to the Q-lattice where it vanishes as an irrelevant deformation. We find that for the linear axion black holes of $[8,27,28]$ that $\bar{\kappa} \rightarrow 0$ as $T \rightarrow 0$. More specifically for the $D=5$ black holes of $[27,28]$, describing anisotropic $N=4$ super Yang-Mills plasma, we find that $\bar{\kappa} \sim T^{7 / 3}$.

The plan of the rest of the paper is as follows. In section 2 we introduce the holographic models and black holes that we will consider in this paper. In section 3 we first calculate $\sigma$ and $\bar{\alpha}$ by deforming the black holes with an applied electric field but with no source for the heat current. This calculation highlights the utility of using a time-like killing vector in the bulk in order to obtain the key expression for the heat current in terms of horizon data. We then complement this analysis by considering a thermal gradient in order to calculate $\bar{\kappa}$ and $\alpha$. Section 4 illustrates our results with some black hole solutions that have been constructed previously. Section 5 concludes. We have three appendices.

\section{The holographic black holes}

\subsection{The Holographic models}

We will mostly focus on holographic models in $D=4$ spacetime dimensions which are dual to $d=3$ CFTs with a global U(1) symmetry. The $D=4$ fields include a metric and a gauge field, which are dual to the stress tensor and the U(1) current of the CFT, respectively. We will also include a real scalar field, $\phi$, and two real "axion" fields, $\chi_{i}$, which are dual to additional scalar operators in the CFT. The action is given by

$$
S=\int d^{4} x \sqrt{-g}\left[R-\frac{1}{2}\left[(\partial \phi)^{2}+\Phi_{1}(\phi)\left(\partial \chi_{1}\right)^{2}+\Phi_{2}(\phi)\left(\partial \chi_{2}\right)^{2}\right]-V(\phi)-\frac{Z(\phi)}{4} F^{2}\right],
$$

which involves four functions, $\Phi_{i}, V$ and $Z$, of the real scalar field $\phi$ and we demand $\Phi_{i}, Z \geq 0$. Also, we have set $16 \pi G=1$. We assume the model admits a unit radius $A d S_{4}$ vacuum with $\phi=0$ (in particular $V(0)=-6$ ) and we shall choose $Z(0)=1$ for convenience. The action is invariant under the global symmetries corresponding to shifts of the axion fields.

\footnotetext{
${ }^{3}$ Some of these IR solutions were also discussed in [7].
} 
For a holographic Q-lattice we are interested in the cases where the fields $\chi_{i}$ are necessarily periodic. These models arise when $\Phi_{i} \sim \phi^{2}$ near $\phi=0$. For example, for a single axion (i.e. setting $\chi_{2}=0$ ), we could consider $\Phi_{1}=\phi^{2}$ and then $\phi, \chi_{1}$ are the norm and phase of a complex scalar field. Furthermore we would choose the mass of this complex field, by choosing $V$, so that the complex field is dual to a relevant operator with dimension $\Delta<3$. A deformation of the CFT by this complex operator with $\chi_{1}$ linear in a spatial direction would necessarily comprise a periodic deformation and hence what we call a holographic lattice. Indeed decomposing the complex field into two real fields, reveals that the construction has two real periodic lattices in the same spatial direction with a phase shift of $\pi / 2$. This was precisely the construction of the anisotropic Q-lattices in [14]. Similarly the models with two $\chi_{i}$ can arise from two complex scalar fields with a $Z_{2}$ symmetry that equates their norms; these constructions lead to isotropic Q-lattices as considered in [6].

Our model also includes other types of black hole solutions where the $\chi_{i}$ are, instead, massless fields, and are dual to marginal operators with $\Delta=3$. These models arise when $\Phi_{i}(0) \neq 0$. For example, the case when $\Phi_{i}=1$ has been considered in [8]. Another case is for a single axion (i.e. setting $\chi_{2}=0$ ) and $\Phi_{1}=e^{2 \phi}$, corresponding to the axion and dilaton of string theory after performing a dimensional reduction of type IIB supergravity on a five-dimensional Einstein space, and anisotropic black holes have been studied in [27-29]. In these cases, the linear axions do not give a periodic deformation of the CFT and hence should not be considered as holographic lattices. Nevertheless, like the Q-lattices, they do incorporate momentum dissipation and have finite DC conductivities.

It is straightforward to generalise our results to other spacetime dimensions. For example, in $D=5$ we could add an additional axion field $\chi_{3}$ along with a coupling $\Phi_{3}(\phi)$, in the obvious way. Our final result for the DC conductivities (see (3.24)) will be written in a way that is also valid for this case too.

\subsection{The black hole backgrounds}

The solutions that we shall consider all lie within the ansatz

$$
\begin{aligned}
d s^{2} & =-U d t^{2}+U^{-1} d r^{2}+e^{2 V_{1}} d x_{1}^{2}+e^{2 V_{2}} d x_{2}^{2}, \\
A & =a d t, \quad \chi_{1}=k_{1} x_{1}, \quad \chi_{2}=k_{2} x_{2},
\end{aligned}
$$

where $U, V_{i}, a$ and $\phi$ are functions of $r$ only. In general the solutions are anisotropic, with $V_{1} \neq V_{2}$, but isotropic solutions with $V_{1}=V_{2}$ are possible when we can choose $k_{1}^{2} \Phi_{1}(\phi)=k_{2}^{2} \Phi_{2}(\phi)$.

We will assume that there is a regular event horizon at $r=r_{+}$with the following expansions

$$
\begin{aligned}
U & \sim 4 \pi T\left(r-r_{+}\right)+\ldots, & V_{i} & \sim V_{i+}+\ldots, \\
a & \sim a_{+}\left(r-r_{+}\right)+\ldots, & \phi & \sim \phi_{+}+\ldots,
\end{aligned}
$$


where $T$ is the temperature of the black hole. Below we will use ingoing EddingtonFinklestein coordinates $(v, r)$ where

$$
v=t+\frac{1}{4 \pi T} \ln \left(r-r_{+}\right) .
$$

As $r \rightarrow \infty$, the location of the $A d S_{4}$ boundary, we assume that

$$
\begin{aligned}
U & \sim r^{2}+\ldots, & e^{2 V_{i}} & \sim r^{2}+\ldots, \\
a & \sim \mu-q r^{-1}+\ldots, & \phi & \sim \lambda r^{\Delta-3}+\ldots,
\end{aligned}
$$

For the case of the Q-lattice, as discussed above, we would demand that $\Delta<3$ and $\lambda$ denotes the strength of the Q-lattice deformation (assuming a standard quantisation for the scalar). For the Q-lattice black holes the axions are periodic, $\chi_{i}=\chi_{i}+2 \pi$, and these UV boundary conditions explicitly break the translation symmetry in a periodic manner. The UV data specifying these black holes is given by $T / \mu, k_{1} / \mu, k_{2} / \mu$ and $\lambda / \mu^{3-\Delta}$. For the case of massless linear axions, as discussed above, $\phi$ can also be massless or absent and the axions are not periodic.

It is useful to obtain a general expression for the electric charge of the black holes in terms of horizon data. The current density $J^{a}=\left(J^{t}, J^{x}, J^{y}\right)$ in the dual field theory has the form

$$
J^{a}=\sqrt{-g} Z(\phi) F^{a r},
$$

where the right hand side is evaluated at the boundary $r \rightarrow \infty$. The only non-zero component of the equation of motion for the gauge-field is in the $t$-direction and can be written $\sqrt{-g} \nabla_{\mu}\left(Z(\phi) F^{\mu t}\right)=\partial_{r}\left(\sqrt{-g} Z(\phi) F^{r t}\right)=0$. Thus we can write

$$
q \equiv J^{t}=e^{V_{1}+V_{2}} Z(\phi) a^{\prime} .
$$

where $q$ is the charge of the black hole and the right hand side can be evaluated at any value of $r$ including $r=r_{+}$. We note that the charge $q$ depends on the UV data of the Q-lattices including the temperature of the black hole.

\section{Calculating the DC conductivities}

\subsection{Calculating $\sigma$ and $\bar{\alpha}$}

In this subsection we recall the derivation of [6] for $\sigma$ and extend it to obtain $\bar{\alpha}$. Specifically, we switch on a constant electric field in the $x_{1}$ direction, with magnitude $E$, and no source for the heat current. For the black holes of interest, this will generate electric and heat currents just in the $x_{1}$ direction, which we will label $J \equiv J^{x_{1}}$ and $Q \equiv Q^{x_{1}}$, respectively. From (1.1) we see that expressions for $\sigma$ and $\bar{\alpha}$ (more precisely $\sigma^{x_{1} x_{1}}$ and $\bar{\alpha}^{x_{1} x_{1}}$ ) can be obtained once we have obtained expressions for $J$ and $Q$. The following derivation will lead to expressions for $\sigma$ and $\bar{\alpha}$ in terms of horizon data. We can obtain the DC conductivity in the $x_{2}$ direction, when they are finite, in an identical manner. 
We consider the following small perturbation about the class of black hole solutions that we considered in the last section

$$
\begin{aligned}
A_{x_{1}} & =-E t+\delta a_{x_{1}}(r), \\
g_{t x_{1}} & =\delta g_{t x_{1}}(r), \\
g_{r x_{1}} & =e^{2 V_{1}} \delta h_{r x_{1}}(r), \\
\chi_{1} & =k_{1} x_{1}+\delta \chi_{1}(r),
\end{aligned}
$$

which one can check is consistent with the linearised equations of motion.

We first show that the linearised gauge-equations of motion imply that the current $J$ is constant and hence obtain an expression in terms of horizon data. Specifically, the only nontrivial component of the gauge equation of motion is the $x_{1}$ component and it can be written in the form $\partial_{r}\left(\sqrt{-g} Z(\phi) F^{r x_{1}}\right)=0$. Using (2.6) we deduce that $J=-\sqrt{-g} Z(\phi) F^{r x_{1}}$ is a constant. Explicitly we have

$$
J=-e^{V_{2}-V_{1}} Z(\phi) U \delta a_{x_{1}}^{\prime}-q e^{-2 V_{1}} \delta g_{t x_{1}},
$$

where the right-hand side can be evaluated at any value of $r$, including at the black hole horizon at $r=r_{+}$.

We next consider the linearised Einstein equations. We find one equation which we can algebraically solve for $\delta h_{r x_{1}}$ giving

$$
\delta h_{r x_{1}}=\frac{E q e^{-V_{1}-V_{2}}}{k_{1}^{2} \Phi_{1}(\phi) U}+\frac{\delta \chi_{1}^{\prime}}{k_{1}},
$$

as well as the following second order ODE

$$
\begin{array}{r}
\delta g_{t x_{1}}^{\prime \prime}+\left(-V_{1}^{\prime}+V_{2}^{\prime}\right) \delta g_{t x_{1}}^{\prime}-\left(2 V_{1}^{\prime}\left(V_{1}^{\prime}+V_{2}^{\prime}\right)+2 V_{1}^{\prime \prime}+\frac{e^{-2 V_{1}} k_{1}^{2} \Phi_{1}(\phi)}{U}\right) \delta g_{t x_{1}} \\
+e^{-V_{1}-V_{2}} q \delta a_{x_{1}}^{\prime}=0 .
\end{array}
$$

We also note that (3.3) implies the equation of motion for $\delta \chi_{1}$.

Before studying these further we first discuss the boundary conditions that must be imposed on the linearised perturbation at infinity and at the black hole horizon. Observe that $\delta \chi_{1}$ only appears in (3.3); we will assume that $\delta \chi_{1}$ is analytic at the black hole event horizon and falls off sufficiently fast at infinity. To ensure that the perturbation is regular as $r \rightarrow r_{+}$i.e. at the black hole horizon, we need to switch from the coordinates $(t, r)$, which are ill-defined there, and employ Eddington-Finklestein coordinates $(v, r)$, with $v$ defined in (2.4). Specifically, in the $(t, r)$ coordinates, the gauge-field will be well defined if we demand that

$$
\delta a_{x_{1}} \sim-\frac{E}{4 \pi T} \ln \left(r-r_{+}\right)+\mathcal{O}\left(r-r_{+}\right),
$$

since then the full gauge-field perturbation in (3.1) has the regular expansion $A_{x_{1}} \sim-E v+$ $\ldots$ in the Eddington-Finklestein coordinates. Notice that near the horizon we have $\delta a_{x_{1}}^{\prime} \sim$ 
$-\frac{E}{U}+\ldots$, a result that we will use below. For the metric perturbation, we see from (3.3) that $\delta h_{r x_{1}}$ is diverging; this can be remedied by demanding that $\delta g_{t x_{1}}$ behaves as

$$
\delta g_{t x_{1}} \sim-\left.\frac{E q e^{V_{1}-V_{2}}}{k_{1}^{2} \Phi_{1}(\phi)}\right|_{r=r_{+}}+\mathcal{O}\left(r-r_{+}\right) .
$$

Notice that this behaviour for $\delta a_{x_{1}}^{\prime}$ and $\delta g_{t x_{1}}$ is consistent with (3.4).

We next consider the behaviour as $r \rightarrow \infty$. From the expression for the gauge-field in (3.1) we see that we have a deformation of an electric field in the $x_{1}$ direction with strength $E$. We also have the fall-off of $\delta a_{x_{1}} \sim J r^{-1}$ which together with (3.5) completely specifies $\delta a_{x_{1}}$. Now, (3.4) has two independent solutions, one of which behaves as $r^{2}$ and the other as $r^{-1}$; in order to have no additional deformations, associated with sources for the heat current, we demand that the coefficient of the former vanishes. Observe that since we have also specified the boundary condition of $\delta g_{t x_{1}}$ at the horizon this completely specifies the solution of (3.4). In addition, notice from (3.3) that for suitable choices of $\delta \chi_{1}$ the fall-off of $\delta h_{r x_{1}}$ as $r \rightarrow \infty$ can be as weak as desired and with vanishing non-normalisable source for $\delta \chi_{1}$.

At this stage we have obtained a perturbation which is well defined in the bulk, including the horizon and we can use it to obtain the DC conductivities $\sigma$ and $\bar{\alpha}$. The DC electric conductivity is given by $\sigma=J / E$. To obtain our final result we now simply evaluate the right hand side of (3.2) at the black hole event horizon $r=r_{+}$. Doing so we obtain the expression found in [6]:

$$
\begin{aligned}
\sigma & =\left[e^{-V_{1}+V_{2}} Z(\phi)+\frac{e^{V_{1}+V_{2}} Z(\phi)^{2}\left(a^{\prime}\right)^{2}}{k_{1}^{2} \Phi_{1}(\phi)}\right]_{r=r_{+}}, \\
& =\left[\frac{Z(\phi) s}{4 \pi e^{2 V_{1}}}+\frac{4 \pi q^{2}}{k_{1}^{2} \Phi_{1}(\phi) s}\right]_{r=r_{+}},
\end{aligned}
$$

where $s=4 \pi e^{V_{1}+V_{2}}$ is the entropy density of the unperturbed black holes.

To obtain a similar expression for $\bar{\alpha}$ we need to obtain an expression for the heat current $Q$ analogous to (3.2). In essence (we also need to use the gauge equation of motion) we need to find a first integral of the equation of motion (3.4). While this can be guessed, the underlying reason ${ }^{4}$ can be clarified by introducing a two-form associated with the Killing vector field $\partial_{t}$. We first observe that if $k$ is an arbitrary Killing vector, and hence $\nabla^{\mu} k^{\nu}=\nabla^{[\mu} k^{\nu]}$, which satisfies $L_{k} F=L_{k} \phi=L_{k} \chi_{i}=0$, then we can define a two-form $G$ by

$$
G^{\mu \nu}=\nabla^{\mu} k^{\nu}+\frac{1}{2} Z(\phi) k^{[\mu} F^{\nu] \sigma} A_{\sigma}+\frac{1}{4}(\psi-2 \theta) Z(\phi) F^{\mu \nu},
$$

where $\psi$ and $\theta$ are defined by $L_{k} A=d \psi$ and $i_{k} F=d \theta$. It has the important property that

$$
\nabla_{\nu} G^{\mu \nu}=-\frac{V}{2} k^{\mu}
$$

when the equations of motion are satisfied. A derivation is provided in appendix A.

\footnotetext{
${ }^{4}$ Note that this would be obscure in the approach to calculate the DC conductivity used in [7-10], where a time dependence of the form $e^{-i \omega t}$ is assumed for the electric field and then later the $\omega \rightarrow 0$ limit is taken.
} 
Focussing now on the Killing vector $k=\partial_{t}$, we consider the $x_{1}$ component of (3.9) to deduce that $\partial_{r}\left(\sqrt{-g} G^{r x_{1}}\right)=0$ and hence that $\sqrt{-g} G^{x_{1} r}$ is a constant. We choose $\theta=-E x_{1}-a$ and $\psi=-E x_{1}$, where we have fixed some free integration constants for convenience, to conclude that at linearised order we can write

$$
\begin{aligned}
Q & \equiv 2 \sqrt{-g} G^{r x_{1}} \\
& =2 \sqrt{-g} \nabla^{r} k^{x_{1}}+a \sqrt{-g} F^{r x_{1}} \\
& =e^{-V_{1}+V_{2}} U^{2}\left(\frac{\delta g_{t x_{1}}}{U}\right)^{\prime}-a J
\end{aligned}
$$

where $Q$ is a constant. Since $Q$ is a constant we can evaluate the right hand side at any value of $r$. In particular, if we evaluate at the boundary $r \rightarrow \infty$, we find that $Q$ is indeed the heat current. The last term is simply $-\mu J$ while, as we explain in appendix B, the first term is $T^{t x_{1}}=r^{5} \bar{T}^{t x_{1}}$ where $\bar{T}^{\mu \nu}$ is the holographic stress tensor of [30] and hence

$$
Q=T^{t x_{1}}-\mu J
$$

We can also evaluate at the black hole horizon and at leading order in $\left(r-r_{+}\right)$we deduce that

$$
Q=-4 \pi T e^{-V_{1}+V_{2}} \delta g_{t x_{1}} .
$$

Then using (3.6) we conclude that $\bar{\alpha}=Q / E$ can be expressed in terms of horizon data of the black holes as

$$
\bar{\alpha}=\left[\frac{4 \pi q}{k_{1}^{2} \Phi_{1}(\phi)}\right]_{r=r_{+}} .
$$

\section{$3.2 \quad$ Calculating $\alpha$ and $\bar{\kappa}$}

We now want to consider perturbations which have a source for the heat current. This will allow us to obtain expressions for $\alpha$ and $\bar{\kappa}$. To do this we will consider the following linearised perturbation about the black hole solutions that we considered in section 2:

$$
\begin{aligned}
A_{x_{1}} & =t \delta f_{1}(r)+\delta a_{x_{1}}(r), \\
g_{t x_{1}} & =t \delta f_{2}(r)+\delta g_{t x_{1}}(r), \\
g_{r x_{1}} & =e^{2 V_{1}} \delta h_{r x_{1}}(r), \\
\chi_{1} & =k_{1} x_{1}+\delta \chi_{1}(r) .
\end{aligned}
$$

We first consider the gauge equations of motion

$$
\partial_{r}\left(\sqrt{-g} Z(\phi) F^{x_{1} r}\right)=0,
$$

where

$$
\sqrt{-g} Z(\phi) F^{x_{1} r}=-e^{V_{2}-V_{1}} Z(\phi)\left[\left(U \delta a_{x_{1}}^{\prime}+a^{\prime} \delta g_{t x_{1}}\right)+t\left(U \delta f_{1}^{\prime}+a^{\prime} \delta f_{2}\right)\right],
$$


and we observe the explicit linear time dependence. We also find that one of the Einstein equations, combined with the gauge equations of motion, is equivalent to the condition that $\tilde{Q}$ is independent of $r$ where

$$
\tilde{Q} \equiv e^{-V_{1}+V_{2}} U^{2}\left[\left(\frac{\delta g_{t x_{1}}}{U}\right)^{\prime}+t\left(\frac{\delta f_{2}}{U}\right)^{\prime}\right]-a \sqrt{-g} Z(\phi) F^{x_{1} r}
$$

The remaining Einstein equation can be solved for $\delta h_{r x_{1}}$ :

$$
\delta h_{r x_{1}}=\frac{q \delta f_{1} e^{-V_{1}-V_{2}}}{k_{1}^{2} \Phi_{1}(\phi) U}+\frac{e^{2 V_{1}}\left(e^{-2 V_{1}} \delta f_{2}\right)^{\prime}}{k_{1}^{2} U \Phi_{1}}+\frac{\delta \chi_{1}^{\prime}}{k_{1}} .
$$

At this point we can observe that if we choose

$$
\begin{aligned}
\delta f_{1} & =-E+\zeta a(r), \\
\delta f_{2} & =-\zeta U(r),
\end{aligned}
$$

for constants $E, \zeta$ then all time dependence drops out of (3.15), (3.17). Equivalently, this choice of $f_{1}, f_{2}$ solves the full linearised equations of motion. We also note that when $\zeta=0$ then we have exactly the same set-up as in the last subsection.

Proceeding with this choice for $\delta f_{1}$ and $\delta f_{2}$ we find that (3.18) now implies the equation of motion for $\delta \chi_{1}$. We also find that defining

$$
\begin{aligned}
J & \equiv-e^{V_{2}-V_{1}} Z(\phi)\left(U \delta a_{x_{1}}^{\prime}+a^{\prime} \delta g_{t x_{1}}\right), \\
Q & \equiv e^{-V_{1}+V_{2}} U^{2}\left(U^{-1} \delta g_{t x}\right)^{\prime}-a J,
\end{aligned}
$$

then both $J$ and $Q$ are constants. By evaluating at $r \rightarrow \infty$, it is clear that $J$ is the current in the $x_{1}$ direction. In appendix B we will explain why $Q$ is the time-independent part of the heat current in the $x_{1}$ direction.

We now analyse regularity conditions at the black hole event horizon. For the gaugefield, as in the last subsection, we again need to impose that

$$
\delta a_{x_{1}} \sim-\frac{E}{4 \pi T} \ln \left(r-r_{+}\right)+\ldots,
$$

where the dots refer to terms analytic in $\left(r-r_{+}\right)$. Again allowing $\delta \chi_{1}$ to be a constant on the horizon, we see from (3.18) that $\delta h_{r x}$ is diverging like $\sim U^{-1}$ at the horizon. By switching to Kruskal coordinates we can ensure regularity of the linearised metric perturbation by choosing the behaviour of $\delta g_{t x}$ to behave near the horizon as

$$
\left.\delta g_{t x} \sim U e^{2 V_{1}} \delta h_{r x}\right|_{r=r_{+}}-\frac{\zeta U}{4 \pi T} \ln \left(r-r_{+}\right)+\ldots .
$$

Observe (unlike in the last subsection) that this implies conditions on the leading and the sub-leading terms in the expansion abut $r=r_{+}$. Remarkably (3.21), (3.22) are consistent with the second order equations for $\delta a_{x_{1}}$ and $\delta g_{t x_{1}}$ with first integrals as in (3.20). Furthermore, we can demand that the fall-off of $\delta g_{t x_{1}}(r) \sim r^{-1}$ and $\delta a_{x_{1}}(r) \sim J r^{-1}$ at infinity, which is consistent with (3.20). To fully specify the perturbation, as in the last subsection, 
we suitably choose $\delta \chi_{1}$ so that the fall-off of $\delta h_{r x_{1}}$ as $r \rightarrow \infty$ is as weak as desired, with vanishing non-normalisable source for $\delta \chi_{1}$. Most importantly, we find that the expansions of the perturbation can be developed with the constants $J, Q$ given at the horizon by

$$
\begin{aligned}
& J=\left[E\left(e^{-V_{1}+V_{2}} Z(\phi)+\frac{e^{V_{1}+V_{2}} Z(\phi)^{2}\left(a^{\prime}\right)^{2}}{k_{1}^{2} \Phi(\phi)}\right)+\zeta \frac{e^{V_{1}+V_{2}} Z(\phi) a^{\prime} U^{\prime}}{k_{1}^{2} \Phi(\phi)}\right]_{r=r_{+}}, \\
& Q=\left[E \frac{e^{V_{1}+V_{2}} Z(\phi) a^{\prime} U^{\prime}}{k_{1}^{2} \Phi(\phi)}+\zeta \frac{e^{V_{1}+V_{2}}\left(U^{\prime}\right)^{2}}{k_{1}^{2} \Phi(\phi)}\right]_{r=r_{+}},
\end{aligned}
$$

and we note that we need to use the background equations of motion to get these expressions.

At this point we have obtained a linearised perturbation about the black holes solutions that is well defined on the black hole horizon and contains pieces that have a linear dependence in time. These time dependent pieces comprise the only holographic sources at the boundary at $r \rightarrow \infty$. We have seen in the previous subsection that when $\zeta=0$ that $E$ parametrizes an electric field deformation. As we explain in appendix B and C, upon setting $E=0$ we can deduce that $\zeta$ parameterises a time dependent source for the heat current. Furthermore, using the results of appendix $\mathrm{C}$, with independent $E, \zeta$ we can now calculate the full DC conductivity matrix, in the $x_{1}$ direction:

$$
\begin{aligned}
\sigma & =\frac{\partial}{\partial E} J=\left[\frac{Z(\phi) s}{4 \pi e^{2 V_{1}}}+\frac{4 \pi q^{2}}{k_{1}^{2} \Phi_{1}(\phi) s}\right]_{r=r_{+}}, \\
\bar{\alpha} & =\frac{1}{T} \frac{\partial}{\partial E} Q=\left[\frac{4 \pi q}{k_{1}^{2} \Phi_{1}(\phi)}\right]_{r=r_{+}}, \\
\alpha & =\frac{1}{T} \frac{\partial}{\partial \zeta} J=\left[\frac{4 \pi q}{k_{1}^{2} \Phi_{1}(\phi)}\right]_{r=r_{+}}, \\
\bar{\kappa} & =\frac{1}{T} \frac{\partial}{\partial \zeta} Q=\left[\frac{4 \pi s T}{k_{1}^{2} \Phi_{1}(\phi)}\right]_{r=r_{+}} .
\end{aligned}
$$

It is a satisfying check that we find $\alpha=\bar{\alpha}$ and hence a symmetric conductivity matrix.

\subsection{Comments}

Although we have carried out the derivation in $D=4$ space-time dimensions, the final expressions (3.24) are also valid in other space-time dimensions. For example, in $D=5$ they are valid when the model (2.1) is generalised to have another axion $\chi_{3}$ with an associated function $\Phi_{3}(\phi)$. The black hole ansatz (2.2) should also be generalised to have $\chi_{3}=k_{3} x_{3}$, with isotropic black holes, with $V_{1}=V_{2}=V_{3}$, only possible when $k_{1}^{2} \Phi_{1}(\phi)=k_{2}^{2} \Phi_{2}(\phi)=$ $k_{3}^{2} \Phi_{3}(\phi)$.

We next note that if one is interested in electrically neutral black holes then the results (3.24) are also valid if we set $q=0$. For example, for the $D=4$ AdS-Schwarzschild black hole we get $\sigma=Z s /\left(4 \pi e^{2 V_{1}}\right)$, for constant $Z$, recovering the result of [3]. The fact that the first term in $\sigma$ is non-zero for neutral black holes with $k_{1}=0$ suggests that, loosely speaking, it is associated with current flow arising from the evolution of charged particlehole pairs, possibly pair created, in an electric field. We should, however, bear in mind that 
there are no quasi-particles. Similarly, the second term, as well as the expressions for $\alpha, \bar{\kappa}$, which diverge as $k_{1} \rightarrow 0$ can be then associated with momentum dissipation. In fact, for general $q$, the first term in $\sigma$ has a simple interpretation as the conductivity in the absence of heat flows, i.e. with $Q=0$. Specifically, we have

$$
\left(\frac{J}{E}\right)_{Q=0} \equiv \sigma-\frac{\alpha \bar{\alpha} T}{\bar{\kappa}}=\left[\frac{Z(\phi) s}{4 \pi e^{2 V_{1}}}\right]_{r=r_{+}}
$$

where the first equality immediately follows from (1.1) and the second from (3.24).

It is interesting to observe that for the class of black holes we are considering we always have the simple relation

$$
\frac{\bar{\kappa}}{\alpha}=\frac{T s}{q} .
$$

Next we recall that $\bar{\kappa}$ is the thermal conductivity at zero electric field. We can also define $\kappa$, the thermal conductivity at zero electric current, a quantity that is more readily measurable. From (1.1) we deduce that $\kappa \equiv \bar{\kappa}-\alpha \bar{\alpha} T / \sigma$ and hence

$$
\kappa=\left[\frac{4 \pi s T e^{2 V_{2}} Z(\phi)}{q^{2}+k_{1}^{2} e^{2 V_{2}} Z(\phi) \Phi_{1}(\phi)}\right]_{r=r_{+}} .
$$

Unlike $\bar{\kappa}$, we see that $\kappa$ is well defined if we set $k_{1}^{2} \Phi_{1}(\phi) \rightarrow 0$. Additional quantities of interest are the ratios of thermal conductivities to electric conductivities. We find

$$
\begin{aligned}
& \bar{L} \equiv \frac{\bar{\kappa}}{\sigma T}=\left[\frac{s^{2}}{q^{2}+k_{1}^{2} e^{2 V_{2}} Z(\phi) \Phi_{1}(\phi)}\right]_{r=r_{+}}, \\
& L \equiv \frac{\kappa}{\sigma T}=\left[\frac{k_{1}^{2} e^{2 V_{2}} s^{2} Z(\phi) \Phi_{1}(\phi)}{\left(q^{2}+k_{1}^{2} e^{2 V_{2}} Z(\phi) \Phi_{1}(\phi)\right)^{2}}\right]_{r=r_{+}} .
\end{aligned}
$$

For Fermi liquids the ability of the quasi particles to transport heat is determined by their ability to transport charge and $L$ is a constant,${ }^{5}$ as encapsulated in the Widemann-Franz law. Deviations from this behaviour is a possible indication of strong interactions. It is also interesting to observe that $\bar{L}$ and $\kappa$ approach finite limits as $k_{1} \rightarrow 0$, while $L$ approaches zero and $\bar{\kappa}$ diverges. We also note that we have the following bound

$$
\bar{L} \leq \frac{s^{2}}{q^{2}},
$$

for all of the black holes we have been considering. Notice that this bound approaches saturation when the second term in the $\sigma$ in (3.24) dominates the first.

In the next subsection we will obtain the thermoelectric DC conductivities for various examples that have been discussed in the literature. It is interesting to obtain the lowtemperature scaling behaviours for the different ground states that can arise. We find examples in which the two terms in $\sigma$ in (3.24) both scale in the same way. We also find

\footnotetext{
${ }^{5}$ In fact when there is purely elastic scattering (either for very low $T$ for $T$ above the Debye temperature where there is elastic phonon scattering) $L=\pi^{2} / 3\left(k_{B} / e\right)^{2}$.
} 
examples, which we might call "pair evolution dominated", in which the first term in (3.24) dominates the second. For both of these classes we find that $\kappa$ and $\bar{\kappa}$ scale in the same way. There is a third class of examples, which we might call "dissipation dominated", in which the second term in (3.24) dominates the first. In this case $\kappa$ and $\bar{\kappa}$ scale in different ways and we approach saturation of (3.29). We will define the state to be metallic if it conducts at $T=0$ and electrically insulating if, instead, $\sigma=0$. Note that for almost all metallic states we will in fact have ${ }^{6} \sigma \rightarrow \infty$ as $T \rightarrow 0$.

We end this section by commenting on the very high temperature behaviour of the conductivities. When $T$ is much bigger than $\mu, k_{i}$ and the scale fixed by the lattice deformation strength $\lambda$, we can approximate the lattice black holes by the $D=4$ AdSSchwarzschild black hole with $U=r^{2}-r_{+}^{3} / r, e^{2 V_{i}} \sim r^{2}$ in (2.2). With $r_{+} \propto T$ we conclude that $s \sim T^{d-1}, q \sim T$. Focussing on the case $\Phi_{i} \sim \phi^{2}$ as $\phi \rightarrow 0$ (the case of periodic axions), we can solve the Laplacian for $\phi$ by first scaling the radial coordinate $r=r_{+} \rho$. At leading order in $r_{+}$there is no dependence on $r_{+}$nor on $k$. We therefore have the solution with behaviour $\phi(\rho=1)$ is a constant independent of $r_{+}$, and, as $\rho \rightarrow \infty$, $\phi(\rho) \sim A \rho^{\Delta-3}=r_{+}^{3-\Delta} A r^{\Delta-3}$, where $A$ is some constant. Now since we want the deformation at infinity to be held fixed when we take the high temperature limit, we should rescale this solution by a factor of $r_{+}^{\Delta-3}$. Thus, at the horizon, $r=r_{+}$, we have $\phi \rightarrow T^{\Delta-3}$ and hence $\left.\left.\Phi_{i}(\phi)\right|_{r=r_{+}} \sim \phi^{2}\right|_{r=r_{+}} \sim T^{2(\Delta-3)}$. We thus conclude that the scaling in $\sigma$ is dominated by the second term in (3.24) (dissipation dominated), and

$$
\sigma \sim T^{2(3-\Delta)}, \quad \alpha \sim \sigma T, \quad \bar{\kappa} \sim \sigma T^{3}, \quad \kappa \sim T^{3},
$$

as $T \rightarrow \infty$. This result is also valid for the case of linear axions with $\Delta=3$. Note, in particular, that when $\Delta<3$ we have a divergent $\sigma$ as $T \rightarrow \infty$. This can be contrasted with the behaviour of the optical conductivity $\sigma(\omega)$ which approaches a constant as $\omega \rightarrow \infty$. For the case of metals, in which the DC conductivity $\sigma$ is diverging at low temperatures, the fact that it also diverges at high temperatures when $\Delta<3$ implies that there will be a minimum conductivity at finite some temperature. This is reminiscent of the Mott-IoffeRegel bound [31, 32], but here, of course, we have no quasi-particles.

\section{Examples}

We illustrate our formulae for the DC conductivity using some AdS black holes that exhibit momentum dissipation which have been discussed in the literature. The simplest are the analytic isotropic solutions of [8] with massless linear axions and so we present these first. We then discuss the anisotropic black holes with massless linear axions of [27, 28] followed by the Q-lattice black holes of $[6,14]$, all of which have been constructed numerically.

\footnotetext{
${ }^{6}$ Note that for arbitrarily small $T$, but $T \neq 0$, we have $\sigma$ is finite and hence so is $J$. Thus, $J$ can be made small by choosing $E$ to be small enough and the set-up is suitable for extracting the linear response.
} 


\subsection{Models with massless axions}

\subsubsection{Analytic isotropic solutions}

A simple class of $D=4$ isotropic black hole solutions for (2.1) with massless axions arises when

$$
\phi=0, \quad \Phi_{i}=1, \quad Z=1, \quad V=-6 .
$$

Indeed the solutions were constructed analytically in $[8,33]$ and are given by

$$
\begin{aligned}
d s^{2} & =-f d t^{2}+\frac{d r^{2}}{f}+r^{2}\left(d x_{1}^{2}+d x_{2}^{2}\right), \\
A & =\mu\left(1-\frac{r_{0}}{r}\right), \\
\chi_{1} & =k x_{1}, \quad \chi_{2}=k x_{2},
\end{aligned}
$$

where $f=r^{2}-k^{2} / 2-m_{0} / r+\mu^{2} r_{0}^{2} / 4 r^{2}$ with $m_{0}=r_{0}^{3}+r_{0}\left(\mu^{2}-2 k^{2}\right) / 4$. The temperature of these black holes is related to $r_{0}$, the radial location of the black hole horizon, via

$$
r_{0}=\frac{2 \pi}{3}\left(T+\sqrt{T^{2}+\frac{3\left(\mu^{2}+2 k^{2}\right)}{16 \pi^{2}}}\right) .
$$

At zero temperature the black hole approach $A d S_{2} \times \mathbb{R}^{2}$ in the far IR. Note that since $\Phi_{i}=1$ the fields $\chi_{i}$ do not have to be periodically identified for these black holes.

Using the formulae that we derived above we deduce that

$$
\sigma=1+\frac{\mu^{2}}{k^{2}}, \quad \alpha=\frac{4 \pi \mu}{k^{2}} r_{0}, \quad \bar{\kappa}=\frac{(4 \pi)^{2}}{k^{2}} \operatorname{Tr}_{0}^{2},
$$

as well as

$$
\bar{L}=\frac{(4 \pi)^{2}}{\left(\mu^{2}+k^{2}\right)} r_{0}^{2}, \quad L=\frac{(4 \pi)^{2} k^{2}}{\left(\mu^{2}+k^{2}\right)^{2}} r_{0}^{2},
$$

which are also valid for the case $\mu=0$. It is interesting to observe that while the electric conductivity $\sigma$ is finite at $T=0$, corresponding to metallic behaviour, the thermal conductivity $\bar{\kappa}$ is going to zero. Notice that for these black holes the two terms in $\sigma$ in (3.24) both scale in the same way at low-temperatures.

\subsubsection{Anisotropic neutral black holes in $D=5$}

We next consider the anisotropic black holes in $D=5$ with a single massless axion field, linear in the $x_{1}$ direction, that were constructed in [27, 28] extending [26]. These black holes are electrically neutral with no gauge field (i.e. $Z=0$ ) and have

$$
\Phi_{i}=e^{2 \phi}, \quad V=-12 .
$$

As $T \rightarrow 0$ the solutions approach Lifshitz solutions in the far IR that are supported by the linear axion. The low temperature behaviour of $\bar{\kappa}$ can be extracted from the finite 
temperature Lifshitz solutions which were found in [26]. The entropy density scales with temperature as $s \sim T^{8 / 3}$ while the scalar scales as $e^{2 \phi} \sim T^{4 / 3}$. Hence we conclude that as $T \rightarrow 0$, in the direction $x_{1}$ of the linear axion,

$$
\bar{\kappa} \sim T^{7 / 3}
$$

Thus these black holes are dual to ground states which are thermally insulating in the direction of the linear axion. In the other spatial directions, $x_{2}$ and $x_{3}$, they are ideal thermal conductors with infinite $\bar{\kappa}$.

For the black holes constructed in $[27,28]$ one can also ask about the electric conductivity. It is natural to consider this question within the context of the bosonic part of $D=5$ minimal supergravity coupled to the axion-dilaton considered in [27, 28]. By extending the arguments in [34] one can show that this theory arises as a consistent Kaluza-Klein truncation of $D=10$ string theory on an internal manifold $M_{5}$ associated with any supersymmetric $A d S_{5} \times M_{5}$ solution of the $D=10$ supergravity theory. Now minimal supergravity has a kinetic term for the Maxwell field combined with a Chern-Simons term. However, for the calculation of the DC conductivity for the class of black holes considered in $[27,28]$, the Chern-Simons term plays no role and we can still use the formula for $\sigma$ given in (3.24) with $q=0$ and $Z=1$. By carrying out a similar analysis as above we find that the low-temperature scaling of the electrical conductivity in the $x_{1}$ direction is given by $\sigma \sim T^{4 / 3}$ while in the $x_{2}$ and $x_{3}$ directions it is given by $\sigma \sim T^{2 / 3}$.

\subsection{Holographic Q-lattices}

Various holographic Q-lattice black hole solutions were constructed in [6, 14], both isotropic and anisotropic. Some of these approach $A d S_{2} \times \mathbb{R}^{2}$ in the IR at $T=0$ while others approach new IR ground states which were independently found in [7].

\subsubsection{Q-lattice black holes with $A d S_{2} \times \mathbb{R}^{2}$ in the IR at $T=0$}

Consider Q-lattice deformations which at zero temperature approach electrically charged $A d S_{2} \times \mathbb{R}^{2}$ solutions in the near horizon limit. As $T \rightarrow 0$, these black holes will have $\left.e^{V_{i}}\right|_{r=r_{+}},\left.Z\right|_{r=r_{+}}, q$ and $s$ all approaching non-zero constant values. On the other hand, for the holographic Q-lattice black holes (unlike the solutions in section 4.1.1 above) as $T \rightarrow 0$ we have $\Phi_{1}(\phi) \rightarrow 0$ near the horizon. More precisely, we have $\Phi_{1}(\phi) \sim T^{2 \Delta\left(k_{1}\right)-2}$ where $\Delta\left(k_{1}\right)>1$ is the dimension of the irrelevant operator arising from perturbations of the scalar field $\phi$ about the $A d S_{2} \times \mathbb{R}^{2}$ background. ${ }^{7}$ Explicit details of this calculation are presented in the examples of $[6,14]$. Thus we immediately deduce that the low temperature scaling of $\sigma$ is dominated by the second term in (3.24) (i.e. is "dissipation dominated") and we have

$$
\sigma \sim T^{2-2 \Delta\left(k_{1}\right)}, \quad \alpha \sim T^{2-2 \Delta\left(k_{1}\right)}, \quad \bar{\kappa} \sim T^{3-2 \Delta\left(k_{1}\right)} .
$$

\footnotetext{
${ }^{7}$ Since there is a renormalisation of length scales from the UV to the IR, the $k_{1}$ appearing in $\Delta\left(k_{1}\right)$ is not the UV lattice momentum $k_{1}$.
} 
The result for $\sigma$ agrees with the arguments of [24], using the memory matrix formalism, which are valid for small lattice perturbations about translationally invariant IR ground states. It is interesting to observe that while $\sigma$ and $\alpha$ will diverge at $T=0, \bar{\kappa}$ will go to zero, if $1<\Delta\left(k_{1}\right)<3 / 2$, a constant, if $\Delta\left(k_{1}\right)=3 / 2$, and diverge if $3 / 2<\Delta\left(k_{1}\right)$. We also find that as $T \rightarrow 0$ we approach a saturation of the bound on $\bar{L}$ given in (3.29):

$$
\bar{L} \rightarrow \frac{s^{2}}{q^{2}}
$$

We also find the following low-temperature scaling behaviours

$$
\kappa \sim T, \quad L \sim T^{2 \Delta\left(k_{1}\right)-2} .
$$

Notice that $\bar{\kappa}$ and $\kappa$ scale in different ways, as do $\bar{L}$ and $L$.

Finally, we note that reference [25] used the memory matrix formalism to argue that these holographic black holes will have, approximately,

$$
\bar{L} \sim \frac{1}{T^{2}} \frac{\chi_{Q P}^{2}}{\chi_{J P}^{2}},
$$

where, in the notation of [25], $\chi$ are static susceptibilities involving the operators for the total momentum $P$, electric current $J$ and heat current $Q$. Similarly, using the results and notation of [25] we conclude that these holographic black holes have, approximately,

$$
\frac{\bar{\kappa}}{\alpha} \sim \frac{\chi_{Q P}}{\chi_{J P}} .
$$

\subsubsection{The Q-lattice black holes of [6]}

Various holographic Q-lattice black hole solutions were discussed in [6]. The most explicit constructions, presented in sections 2 and 3 of [6], involved anisotropic lattices with a single axion field i.e. $\Phi_{2}=\chi_{2}=0$ and specific choices for $\Phi_{1}, V$ and $Z$ that involved a free parameter $\gamma$ with $-1<\gamma$. Depending on the value of $\gamma$ it was shown that there can be metal-insulator as well as metal-metal transitions driven by the strength of the holographic lattice deformation.

In some cases at $T=0$ the black holes approach $A d S_{2} \times \mathbb{R}^{2}$ in the far IR, with nonvanishing entropy density. In other cases the $T=0$ black holes approach new ground states, breaking translation invariance, which were presented in section 2 of [6] (and also in [7]). By analysing the small temperature behaviour of these ground states by heating them up (i.e. by constructing small black hole solutions) some calculation reveals the following scaling behaviours as $T / \mu \rightarrow 0$ :

$$
\sigma \sim T^{\frac{(1+\gamma)(3-\gamma)}{9+2 \gamma+\gamma^{2}}}, \quad \alpha \sim T^{\frac{4(1+\gamma)}{9+2 \gamma+\gamma^{2}}}, \quad \kappa, \bar{\kappa} \sim T^{\frac{2\left(7+4 \gamma+\gamma^{2}\right)}{9+2 \gamma+\gamma^{2}}}, \quad L, \bar{L} \sim T^{\frac{2(1+\gamma)^{2}}{9+2 \gamma+\gamma^{2}}} .
$$

Note that for $\sigma$, both terms in (3.24) scale in the same way. In addition $q$ scales like $T^{0}$ as does the other term appearing in the denominator of (3.27) and (3.28). By considering $\sigma$ we deduce that for $-1<\gamma<3$ the ground states are insulators, while if $3 \leq \gamma$ they are 
metals. Observe that for $-1<\gamma$ the exponents in $\alpha$ and $\bar{L}, L$ are greater than zero, while the exponent for $\bar{\kappa}, \kappa$ is greater than one. The fact that $\bar{\kappa}, \kappa \rightarrow 0$ at $T=0$ says that a heat gradient does not give rise to transport. On the other hand if $3 \leq \gamma$ there is transport of charge. This indicates that the latter transport can be loosely thought of as due to the evolution of charged particle/anti-particle pairs (even though these metals are not "pair creation dominated" as defined below (3.29)).

In section 4 of [6] (and also in [7]) a different class of metallic and insulating ground state solutions, isotropic in the spatial directions, were constructed which depended on three constants $c, \alpha$ and $\gamma$. For reasons that were explained in [6], it was natural to focus on the range

$$
2 \leq \alpha<\sqrt{4+c}, \quad \gamma \geq \alpha-4 .
$$

The far IR of the ground states are electrically neutral solutions to the equations of motion with vanishing gauge-field, but the conditions (4.14) imply that one can shoot out with an irrelevant or marginal operator to match on to the UV with $\mu \neq 0$. By analysing the small black hole solutions, we find the following low temperature behaviour. We find that $q$ is again independent of $T$ and that

$$
\sigma \sim T^{-\frac{2(\alpha-2) \gamma}{4+c-\alpha^{2}}}, \quad \alpha \sim T^{\frac{4(\alpha-2)}{4+c-\alpha^{2}}}, \quad \kappa, \bar{\kappa} \sim T^{\frac{4+c-4 \alpha+\alpha^{2}}{4+c-\alpha^{2}}}, \quad L, \bar{L} \sim T^{\frac{2(\alpha-2)(\gamma+\alpha)}{4+c-\alpha^{2}}} .
$$

The first term appearing in $\sigma$ in (3.24) now dominates the second term (i.e. they are "pairevolution" dominated). Similarly the second term in the denominators of (3.27), (3.28) dominate the first. By considering $\sigma$ we deduce that for $\gamma \geq 0$ or $\alpha=2$ the ground states are electrical insulators, while if $3 \leq \gamma$ they are metals. When $\alpha \neq 2$ we always have $\kappa, \bar{\kappa} \rightarrow 0$ at $T=0$ which again indicates that for the metallic states the transport in an electric field might be viewed as arising from evolution of charged particle-hole pairs. A special case is when $\alpha=2$ where both $\sigma$ and $\kappa, \bar{\kappa}$ go to a constant at $T=0$. However, it is not yet clear if solutions which asymptote to $A d S_{4}$ exist when $\alpha=2$.

\section{Final comments}

The main result of this paper is an expression for the thermoelectric DC conductivity matrix for a class of asymptotically AdS black holes in terms of black hole horizon data. To achieve this we introduced sources for the electric and heat currents that are linear in time. The full linearised perturbation also contains a time independent piece and we showed that it was possible to obtain expressions for the time independent pieces of the electric and heat currents as total derivatives in the radial direction which could then be written in terms of horizon data. For the electric current this step arises directly from the gauge equations of motion while for the heat current we saw that it arises from the existence of time-like Killing vectors for the time independent perturbation. The final step to obtain the conductivity was to ensure regularity of the perturbation at the black hole horizon.

We obtained some general conditions on the conductivity including (1.2). For small lattice deformations, for which the memory matrix applies, these can be recast as (1.3). 
Using our new results we obtained the thermoelectric DC conductivity for several explicit examples finding some interesting results. For example, the zero temperature ground states can be dissipation dominated, when the second term in $\sigma$ in (3.24) dominates the first, and then $\bar{\kappa}$ and $\kappa$ scale in different ways. These include examples in which the ground states approach in the IR, $A d S_{2} \times \mathbb{R}^{2}$ deformed by irrelevant operators. We also found that the isotropic and the anisotropic ground states found in [6] which break translation invariance, are all thermal insulators despite the fact that they can be electrically insulating or conducting. The isotropic ground states are pair-evolution dominated, with the first term in $\sigma$ in (3.24) dominating the second, while for the anisotropic ground states the two terms are equally important at low temperatures.

The black holes we have considered are homogeneous in the holographic directions. While they can be spatially both isotropic and anisotropic, the conductivity matrix is diagonal and furthermore the models have $\alpha=\bar{\alpha}$ because of the underlying time-reversal invariance. However, our approach can be extended to more general set-ups as we will explain in [23].

We end by briefly commenting on two papers that appeared very recently. In [35] black holes involving a scalar field and interpolating between two $A d S_{4}$ geometries were constructed. These black holes fall within the class of solutions considered here and in [6] and have zero charge density, $q=0$. In particular, the formula for the DC conductivity given in [6], which is valid in the $q=0$ limit, implies that $\sigma \sim Z(\phi)$. The model of [35] is arranged so that $Z(\phi) \rightarrow 0$ in the far IR, leading to a vanishing electrical conductivity, with a power law behaviour in $T$. Clearly the precise power will depend not only on the IR scaling dimension of the operator dual to $\phi$, but also on the choice of $Z$. Note, also, that this model has no mechanism for momentum dissipation and hence there is a delta function in the thermal conductivity. It is also worth noting that since $Z \rightarrow 0$ in the IR, it will not be possible to add charge to these systems and furthermore quantum corrections will be important.

The second paper, [36], calculates the thermoelectric response in the context of massive gravity, developing the work of $[9,10]$. The optical conductivities were calculated numerically, and from this it was possible to extrapolate some behaviour of the DC conductivity. Our results here indicate that it should also be possible to obtain analytic results for the DC thermoelectric conductivities for the models considered in [36].

\section{Acknowledgments}

We thank Mike Blake and David Tong for helpful discussions. The work is supported by STFC grant ST/J0003533/1, EPSRC programme grant EP/K034456/1 and also by the European Research Council under the European Union's Seventh Framework Programme (FP7/2007-2013), ERC Grant agreements STG 279943 and ADG 339140. 


\section{A Killing vector identity}

Suppose that $k$ is a Killing vector. We have, by definition, $\nabla_{(\mu} k_{\nu)}=0$ and hence $\nabla^{\mu} k^{\nu}=$ $\nabla^{[\mu} k^{\nu]}$. In addition we will suppose that we have $L_{k} F=L_{k} \phi=L_{k} \chi_{i}=0$. We will work in $D$ spacetime dimensions. We first observe that using the Bianchi identity we have $\left(i_{k} d+d i_{k}\right) F=d\left(i_{k} F\right)=0$ and hence

$$
k^{\mu} F_{\mu \nu}=\nabla_{\nu} \theta
$$

for some function $\theta$. Using this and the equation of motion for the gauge-field, $\nabla_{\mu}\left(Z(\phi) F^{\mu \nu}\right)=0$, we deduce that

$$
k^{\rho} Z(\phi) F_{\rho \mu}^{2}=\nabla_{\rho}\left(\theta Z(\phi) F_{\mu}{ }^{\rho}\right) .
$$

By writing $\left(L_{k} F\right)_{\mu \nu}=k^{\mu} \nabla_{\mu} F_{\nu \rho}+\nabla_{\nu} k^{\mu} F_{\mu \rho}+\nabla_{\rho} k^{\mu} F_{\nu \mu}=0$ we can similarly show

$$
k^{\mu} Z(\phi) F^{2}=4 \nabla_{\rho}\left(Z(\phi) k^{[\mu} F^{\rho] \nu} A_{\nu}\right)+2 \nabla_{\rho}\left(Z(\phi) F^{\mu \rho} \psi\right),
$$

where $\psi$ is defined via $L_{k} A=d \psi$.

We can now calculate

$$
\begin{aligned}
\nabla_{\mu}\left(\nabla^{\nu} k^{\mu}\right) & =R_{\mu}^{\nu} k^{\mu}, \\
& =\frac{V}{D-2} k^{\nu}+\frac{1}{2} k^{\mu} Z(\phi) F_{\mu}^{2 \nu}-\frac{1}{4(D-2)} k^{\nu} Z(\phi) F^{2},
\end{aligned}
$$

where we used the Einstein equations and $L_{k} \chi_{i}=0$ to get the second line. We thus conclude that when the equations of motion are satisfied we have

$$
\nabla_{\mu} G^{\mu \nu}=-\frac{V}{D-2} k^{\nu}
$$

where the two-form $G$ is given by

$$
G^{\mu \nu}=\nabla^{\mu} k^{\nu}+\frac{1}{D-2} Z(\phi) k^{[\mu} F^{\nu] \sigma} A_{\sigma}+\frac{1}{2(D-2)}(\psi-(D-2) \theta) Z(\phi) F^{\mu \nu} .
$$

Note that we have

$$
\partial_{\mu}\left(\sqrt{-g} G^{\mu \nu}\right)=-\frac{V}{D-2} \sqrt{-g} k^{\nu},
$$

and if we have $k=\partial_{t}$ then we have the right hand side is zero unless $\nu=t$.

\section{B Holographic renormalisation and the heat current}

The action (2.1) should be supplemented with suitable boundary terms. For illustration we assume that we are considering a holographic Q-lattice and in the $A d S_{4}$ vacuum the field $\phi$ is dual to a relevant operator with dimension $\Delta=2$. Then, for the solutions of interest, we should use

$$
S_{c t}=\int d^{3} x \sqrt{-\gamma}\left(2 K-4-\frac{1}{2} \phi^{2}+\ldots\right)
$$


where $K$ is the trace of the extrinsic curvature and we have neglected additional terms involving the Ricci scalar which we don't need. Following [30], the stress tensor and the current are given by

$$
\begin{aligned}
\frac{1}{2} \bar{T}^{\mu \nu} & =-\left[K^{\mu \nu}-K \gamma^{\mu \nu}+\frac{1}{2}\left(4+\frac{1}{2} \phi^{2}\right) \gamma^{\mu \nu}+\ldots\right], \\
\bar{J}^{\nu} & =-n_{\mu} F^{\mu \nu} Z(\phi),
\end{aligned}
$$

where the neglected term in the first line involves the components of the Einstein tensor for the boundary metric which won't contribute, and the right hand side of both lines are evaluated at the boundary $r \rightarrow \infty$. Note that in our conventions, on-shell we have

$$
\delta S=\int d^{3} x \sqrt{-\gamma}\left(\frac{1}{2} \bar{T}^{\mu \nu} \delta \gamma_{\mu \nu}+\bar{J}^{\mu} \delta A_{\mu}\right) .
$$

For the black hole backgrounds we have $n_{\mu}=\left(0,1 / U^{1 / 2}, 0,0\right)$ and we find that

$$
\begin{aligned}
\bar{T}^{t t} & =U^{-1}\left(4+\frac{1}{2} \phi^{2}-2 U^{1 / 2}\left(V_{1}^{\prime}+V_{2}^{\prime}\right)\right), \\
\bar{T}^{x x} & =e^{-2 V_{1}}\left(-4-\frac{1}{2} \phi^{2}+U^{-1 / 2} U^{\prime}+2 U^{1 / 2} V_{2}^{\prime}\right), \\
\bar{T}^{y y} & =e^{-2 V_{2}}\left(-4-\frac{1}{2} \phi^{2}+U^{-1 / 2} U^{\prime}+2 U^{1 / 2} V_{1}^{\prime}\right), \\
\bar{J}^{t} & =\frac{Z(\phi)}{U^{1 / 2}} a^{\prime} .
\end{aligned}
$$

As $r \rightarrow \infty$ we have $\bar{T}^{\mu \nu} \sim r^{-5}$ and $\bar{J}^{\mu} \sim r^{-3}$ so it is convenient to define

$$
T^{\mu \nu}=r^{5} \bar{T}^{\mu \nu}, \quad J^{\mu}=r^{3} \bar{J}^{\mu} .
$$

Note that this is consistent with the definition of $J^{\mu}$ given in (2.6).

We next consider the perturbation (3.14) about the background, but with a general $g_{t x_{1}}(t, r)$ for the moment, finding

$$
\bar{T}^{t x_{1}}=\frac{e^{-2 V_{1}}}{U^{1 / 2}}\left(g_{t x_{1}}(t, r)\left[2 V_{2}^{\prime}-U^{-1 / 2}\left(4+\frac{\phi^{2}}{2}\right)\right]+\partial_{r} g_{t x_{1}}(t, r)\right),
$$

It will be convenient, shortly, to note that ${ }^{8}$

$$
U^{1 / 2} e^{V_{1}+V_{2}}\left(U \bar{T}^{t x_{1}}-g_{t x_{1}}(t, r) \bar{T}^{x_{1} x_{1}}\right)=e^{-V_{1}+V_{2}} U^{2} \partial_{r}\left(\frac{g_{t x_{1}}(t, r)}{U}\right) .
$$

We now consider the particular linearised time-dependence for the perturbation given in (3.14). The most important pieces of the perturbation of relevance here are

$$
\begin{aligned}
A_{x_{1}} & =-t E+t \zeta a(r)+\delta a_{x_{1}}(r), \\
g_{t x_{1}}(t, r) & =-t \zeta U+\delta g_{t x_{1}}(r),
\end{aligned}
$$

\footnotetext{
${ }^{8}$ Observe, in passing, the similarity of the left hand side with equation (15) of [30].
} 
with $\delta a_{x_{1}}, \delta g_{t x_{1}}(r) \sim r^{-1}$ as $r \rightarrow \infty$. We are interested in obtaining the expectation values for the $J^{x_{1}}$ and $T^{t x_{1}}$. Now in the text we showed the time-dependent sources given in (3.14) give rise to a time-independent expression for $J^{x_{1}}$ given in (3.20). However we obtain a time-dependent source for $T^{t x_{1}}$. Explicitly, from (B.6) we immediately obtain

$$
\begin{aligned}
\bar{T}^{t x_{1}} & =e^{-2 V_{1}} U^{-1 / 2}\left(\delta g_{t x_{1}}(r)\left[2 V_{2}^{\prime}-U^{-1 / 2}\left(4+\frac{\phi^{2}}{2}\right)\right]+\partial_{r} \delta g_{t x_{1}}(r)\right)-\zeta t \bar{T}^{x_{1} x_{1}} \\
& \equiv \bar{T}_{0}^{t x_{1}}-\zeta t \bar{T}^{x_{1} x_{1}}
\end{aligned}
$$

Returning now to (B.7) and substituting in (B.8) we find that all of the time dependence drops out and hence we can conclude that

$$
U^{1 / 2} e^{V_{1}+V_{2}}\left(U \bar{T}_{0}^{t x_{1}}-\delta g_{t x_{1}}(r) \bar{T}^{x_{1} x_{1}}\right)=e^{-V_{1}+V_{2}} U^{2} \partial_{r}\left(U^{-1} \delta g_{t x_{1}}(r)\right) .
$$

Evaluating both sides at $r \rightarrow \infty$ we deduce that

$$
r^{5} \bar{T}_{0}^{t x_{1}}=\left.e^{-V_{1}+V_{2}} U^{2} \partial_{r}\left(U^{-1} \delta g_{t x_{1}}(r)\right)\right|_{r \rightarrow \infty} .
$$

Recalling the expression for $Q$ given in (3.20), we deduce that

$$
T^{t x_{1}}-\mu J^{x_{1}}=Q-\zeta t T^{x_{1} x_{1}}
$$

We now ask how this time dependent response fits our expectations. The sources in our perturbation (B.8) are time dependent. After substituting into (B.3) we deduce that

$$
\delta S=\int d^{3} x\left[\left(T^{t x_{1}}-\mu J^{x_{1}}\right)(-\zeta t)+J^{x_{1}}(-E t)\right]
$$

In particular, this shows that $-\zeta$ is parametrizing a time dependent source for the operator $\left(T^{t x_{1}}-\mu J^{x_{1}}\right)$. In appendix $\mathrm{C}$ we show that sources which are linear in time give a response, captured in the expectation value of the operators, that contains, in general, a piece that is linear in time and a time-independent piece - see equation (C.7). The time dependent piece is determined by the associated Greens function matrix at zero frequency and defines a static susceptibility. Since the only linear time dependence appears in (B.11) we deduce that the only non-zero component of this matrix is the $Q^{x_{1}}, Q^{x_{1}}$ component, where $Q^{x_{1}}=$ $T^{t x_{1}}-\mu J^{x_{1}}$, with $\tilde{G}_{Q^{x_{1}} Q^{x_{1}}}(0)=T^{x_{1} x_{1}}$.

Our principle interest is the DC conductivity, as defined in terms of the spectral weight in (C.6). From (C.7) we see that this is captured by the time-independent pieces of the expectation values. Using the results of this appendix and those in the text, then leads to the prescription that we employed in (3.24).

\section{B.1 A complementary point of view}

Let us consider the following coordinate transformation on the boundary:

$$
\begin{aligned}
t & =\bar{t}-\zeta \bar{t} \bar{x}_{1}, \\
x_{1} & =\bar{x}_{1} .
\end{aligned}
$$


At linearised order we find that in these co-ordinates we have

$$
\begin{aligned}
& T^{\overline{t t}}=\left(1+2 \zeta \bar{x}_{1}\right) T^{t t}, \quad T^{\bar{x}_{1} \bar{x}_{1}}=T^{x_{1} x_{1}}, \quad T^{\bar{t}_{1}}=T_{0}^{t x_{1}}, \\
& J^{\bar{t}}=\left(1+\zeta \bar{x}_{1}\right) J^{t}, \quad J^{\bar{x}_{1}}=J^{x_{1}},
\end{aligned}
$$

and, in particular, the time-dependence has dropped out of the expectation values.

Let us now see how this coordinate transformation effects the asymptotic behaviour of the bulk fields at $r \rightarrow \infty$ :

$$
\begin{aligned}
d s^{2} & =-U\left(1-2 \zeta \bar{x}_{1}\right) d \bar{t}^{2}+r^{2} d \bar{x}_{1}^{2} \ldots, \\
A & =a\left(1-\zeta \bar{x}_{1}\right) d \bar{t}-\bar{t} E d \bar{x}_{1}+\ldots .
\end{aligned}
$$

When $E=0$ we can identify $-\zeta$ as a source for a static thermal gradient in the $\bar{x}_{1}$ direction, $\left(\partial_{\bar{x}_{1}} T\right) / T$, with no source for the electric field. This again leads to the prescription that we employed in (3.24).

\section{DC transport from linear sources in time}

We consider a set of sources $s_{A}(t)$ associated with a set of operators $\phi_{A}$. At the level of linear response we have

$$
\langle\phi(t)\rangle_{B}=\int d t^{\prime} G_{B A}\left(t-t^{\prime}\right) s_{A}\left(t^{\prime}\right),
$$

where $G$ is the associated retarded Green's function and we will define the Fourier transform as

$$
G_{B A}(t)=\frac{1}{2 \pi} \int d \omega \tilde{G}_{B A}(\omega) e^{-i \omega t} .
$$

We now examine the implications of sources linear in time $s_{A}(t)=c_{A} t$ leading to

$$
\langle\phi(t)\rangle_{B}=\frac{1}{2 \pi} \int d t^{\prime} d \omega e^{-i \omega\left(t-t^{\prime}\right)} t^{\prime} \tilde{G}_{B A}(\omega) c_{A} .
$$

Using

$$
\int d t^{\prime} e^{i \omega t^{\prime}} t^{\prime}=-2 \pi i \delta^{\prime}(\omega)
$$

we obtain

$$
\begin{aligned}
\langle\phi(t)\rangle_{B} & =i \partial_{\omega}\left(e^{-i \omega t} \tilde{G}_{B A}(\omega)\right)_{\omega=0} c_{A} \\
& =\left(t \tilde{G}_{B A}(0)+i \tilde{G}_{B A}^{\prime}(0)\right) c_{A} .
\end{aligned}
$$

We next define the conductivity matrix $\sigma_{B A}$ as the zero-frequency limit of the spectral weight:

$$
\sigma_{B A}=\lim _{\omega \rightarrow 0} \operatorname{Im} \frac{\tilde{G}_{B A}(\omega)}{\omega} .
$$

Using the fact that the real and the imaginary parts of the Green's function are even and odd functions of $\omega$, respectively, we can rewrite (C.5) as

$$
\langle\phi(t)\rangle_{B}=\left(t \tilde{G}_{B A}(0)-\sigma_{B A}\right) c_{A} .
$$


Open Access. This article is distributed under the terms of the Creative Commons Attribution License (CC-BY 4.0), which permits any use, distribution and reproduction in any medium, provided the original author(s) and source are credited.

\section{References}

[1] S.A. Hartnoll, Lectures on holographic methods for condensed matter physics, Class. Quant. Grav. 26 (2009) 224002 [arXiv:0903.3246] [INSPIRE].

[2] C.P. Herzog, Lectures on Holographic Superfluidity and Superconductivity, J. Phys. A 42 (2009) 343001 [arXiv:0904.1975] [INSPIRE].

[3] N. Iqbal and H. Liu, Universality of the hydrodynamic limit in AdS/CFT and the membrane paradigm, Phys. Rev. D 79 (2009) 025023 [arXiv:0809.3808] [INSPIRE].

[4] G. Policastro, D.T. Son and A.O. Starinets, The Shear viscosity of strongly coupled $N=4$ supersymmetric Yang-Mills plasma, Phys. Rev. Lett. 87 (2001) 081601 [hep-th/0104066] [INSPIRE].

[5] P. Kovtun, D.T. Son and A.O. Starinets, Viscosity in strongly interacting quantum field theories from black hole physics, Phys. Rev. Lett. 94 (2005) 111601 [hep-th/0405231] [INSPIRE].

[6] A. Donos and J.P. Gauntlett, Novel metals and insulators from holography, JHEP 06 (2014) 007 [arXiv: 1401.5077] [INSPIRE].

[7] B. Goutéraux, Charge transport in holography with momentum dissipation, JHEP 04 (2014) 181 [arXiv:1401.5436] [INSPIRE].

[8] T. Andrade and B. Withers, A simple holographic model of momentum relaxation, JHEP 05 (2014) 101 [arXiv:1311.5157] [INSPIRE].

[9] R.A. Davison, Momentum relaxation in holographic massive gravity, Phys. Rev. D 88 (2013) 086003 [arXiv: 1306 .5792] [INSPIRE].

[10] M. Blake and D. Tong, Universal Resistivity from Holographic Massive Gravity, Phys. Rev. D 88 (2013) 106004 [arXiv:1308.4970] [INSPIRE].

[11] R.A. Davison, K. Schalm and J. Zaanen, Holographic duality and the resistivity of strange metals, Phys. Rev. B 89 (2014) 245116 [arXiv:1311.2451] [INSPIRE].

[12] M. Blake, D. Tong and D. Vegh, Holographic Lattices Give the Graviton an Effective Mass, Phys. Rev. Lett. 112 (2014) 071602 [arXiv:1310.3832] [inSPIRE].

[13] M. Blake and A. Donos, Quantum Critical Transport and the Hall Angle, arXiv:1406.1659 [INSPIRE].

[14] A. Donos and J.P. Gauntlett, Holographic Q-lattices, JHEP 04 (2014) 040 [arXiv: 1311.3292] [INSPIRE].

[15] A. Donos and S.A. Hartnoll, Interaction-driven localization in holography, Nature Phys. 9 (2013) 649 [arXiv:1212.2998] [INSPIRE].

[16] N. Iizuka, S. Kachru, N. Kundu, P. Narayan, N. Sircar et al., Bianchi Attractors: A Classification of Extremal Black Brane Geometries, JHEP 07 (2012) 193 [arXiv:1201.4861] [INSPIRE]. 
[17] A. Donos and J.P. Gauntlett, Helical superconducting black holes, Phys. Rev. Lett. 108 (2012) 211601 [arXiv: 1203.0533] [INSPIRE].

[18] G.T. Horowitz, J.E. Santos and D. Tong, Optical Conductivity with Holographic Lattices, JHEP 07 (2012) 168 [arXiv:1204.0519] [INSPIRE].

[19] G.T. Horowitz, J.E. Santos and D. Tong, Further Evidence for Lattice-Induced Scaling, JHEP 11 (2012) 102 [arXiv:1209.1098] [InSPIRE].

[20] G.T. Horowitz and J.E. Santos, General Relativity and the Cuprates, arXiv:1302.6586 [INSPIRE].

[21] Y. Ling, C. Niu, J.-P. Wu and Z.-Y. Xian, Holographic Lattice in Einstein-Maxwell-Dilaton Gravity, JHEP 11 (2013) 006 [arXiv:1309.4580] [INSPIRE].

[22] P. Chesler, A. Lucas and S. Sachdev, Conformal field theories in a periodic potential: results from holography and field theory, Phys. Rev. D 89 (2014) 026005 [arXiv:1308.0329] [INSPIRE].

[23] A. Donos and J.P. Gauntlett, The thermoelectric properties of inhomogeneous holographic lattices, arXiv:1409.6875 [INSPIRE].

[24] S.A. Hartnoll and D.M. Hofman, Locally Critical Resistivities from Umklapp Scattering, Phys. Rev. Lett. 108 (2012) 241601 [arXiv:1201.3917] [INSPIRE].

[25] R. Mahajan, M. Barkeshli and S.A. Hartnoll, Non-Fermi liquids and the Wiedemann-Franz law, Phys. Rev. B 88 (2013) 125107 [arXiv: 1304.4249] [INSPIRE].

[26] T. Azeyanagi, W. Li and T. Takayanagi, On String Theory Duals of Lifshitz-like Fixed Points, JHEP 06 (2009) 084 [arXiv: 0905. 0688] [INSPIRE].

[27] D. Mateos and D. Trancanelli, The anisotropic $N=4$ super Yang-Mills plasma and its instabilities, Phys. Rev. Lett. 107 (2011) 101601 [arXiv:1105.3472] [INSPIRE].

[28] D. Mateos and D. Trancanelli, Thermodynamics and Instabilities of a Strongly Coupled Anisotropic Plasma, JHEP 07 (2011) 054 [arXiv:1106.1637] [INSPIRE].

[29] L. Cheng, X.-H. Ge and S.-J. Sin, Anisotropic plasma at finite U(1) chemical potential, JHEP 07 (2014) 083 [arXiv: 1404.5027] [INSPIRE].

[30] V. Balasubramanian and P. Kraus, A Stress tensor for Anti-de Sitter gravity, Commun. Math. Phys. 208 (1999) 413 [hep-th/9902121] [InSPIRE].

[31] O. Gunnarsson, M. Calandra and J. Han, Colloquium: Saturation of electrical resistivity, Rev. Mod. Phys. 75 (2003) 1085 [cond-mat/0305412].

[32] N. E. Hussey, K. Takenaka and H. Takagi, Universality of the Mott-Ioffe-Regel limit in metals, Phil. Mag. 84 (2004) 2847 [cond-mat/0404263].

[33] Y. Bardoux, M.M. Caldarelli and C. Charmousis, Shaping black holes with free fields, JHEP 05 (2012) 054 [arXiv: 1202 .4458] [INSPIRE].

[34] J.P. Gauntlett and O. Varela, Consistent Kaluza-Klein reductions for general supersymmetric AdS solutions, Phys. Rev. D 76 (2007) 126007 [arXiv:0707.2315] [INSPIRE].

[35] E. Mefford and G.T. Horowitz, Simple holographic insulator, Phys. Rev. D 90 (2014) 084042 [arXiv: 1406.4188] [INSPIRE].

[36] A. Amoretti, A. Braggio, N. Maggiore, N. Magnoli and D. Musso, Thermo-electric transport in gauge/gravity models with momentum dissipation, JHEP 09 (2014) 160 [arXiv: 1406.4134] [INSPIRE]. 